\title{
A method to tune the island size and improve the uniformity for the in situ formation of InGaAs quantum dots on GaAs
}

\author{
Shou-Zen Chang ${ }^{1}$, Tien-Chih Chang, Si-Chen Lee * \\ Department of Electrical Engineering, National Taiwan University, Taipei, Taiwan, ROC \\ Received 12 December 1994; accepted for publication 3 February 1995
}

\begin{abstract}
The use of island formation during the epitaxy of $\operatorname{In}_{x} \mathrm{Ga}_{1-x}$ As on GaAs can be applied to the in situ formation of quantum dots. Adjusting the alloy composition toward a larger degree of cation disorder (Ga and In intermixing) can decrease the island size and improve the island uniformity simultaneously. Under the same deposition conditions with a thickness of 15 monolayers, the dot size can be tuned from $125 \times 100$ to $30 \times 28 \mathrm{~nm}^{2}$ in lateral dimensions as the In composition changes from 1 to 0.5 . Among all conditions, $\mathrm{In}_{0.5} \mathrm{Ga}_{0.5}$ As dots show a high density of $5 \times 10^{10} \mathrm{~cm}^{-2}$ and exhibit the best uniformity. These small-sized and dislocation-free islands are of interest for the formation of high-quality quantum dots.
\end{abstract}

\section{Introduction}

The development of advanced techniques to fabricate low-dimensional structures such as quantum wells and dots has attracted much attention and effort, because these microstructures can modify the optical and transport properties of conventional devices. Quantum dots having controllable dimensions, good uniformity and low process-induced damages are favored. Many fabrication techniques have been developed, including the electron-beam lithography following by ion milling [1], the selective epitaxy on patterned masks [2,3], and the use of the strain-induced field to confine carriers [4]. Alternative in situ

\footnotetext{
" Corresponding author. Fax: +886-2-3638247.

' Now at Communication Development Office, Department of National Defence, Hsin-Tien, Taipei, Taiwan, ROC.
}

epitaxial methods, such as the droplet epitaxy which forms $\mathrm{Ga}$ and In droplets in the molecular beam epitaxy (MBE) growth [5], and the epitaxy growth on substrates with high index [6], are also proposed. Since the in situ growth method is a simpler process which does not require to expose the dots to air, has no limitation on the dot dimension usually constrained by the resolution of lithography, and causes almost no process-induced damage to the dots, it becomes very attractive.

It is known that in the heteroepitaxy of highly lattice-mismatched systems, the growth follows the Stranski-Krastanow mode in which the epitaxy proceeds initially layer-by-layer for a few monolayers (MLs) and subsequently converts to islands [7]. During this process, the formation of dislocation-free coherent islands is energetic favorable rather than the direct generation of dislocations [8-10]. For compound semiconductor materials, the utilization of the 
island growth mode in the epitaxy to develop the in situ fabrication of quantum dots has been reported recently, for example $\operatorname{In}_{0.5} \mathrm{Ga}_{0.5}$ As and InAs islands, respectively, grown on GaAs [11,12], and InP islands embedded in the InGaP layers which are grown on GaAs $[13,14]$. Using this method, islands with diameters about $30 \mathrm{~nm}$ in the lateral dimension were formed, much smaller than the ones fabricated through the lithography method $[11,12]$. Intense photoluminescence signals from these dots were also obtained [11]. The main challenge to this epitaxial method $[11,13,14]$ is the capability of controlling the dot size and uniformity. The general method employed to tune the island size is to change the deposition time [12] or conditions, such as changing the off-angles of the substrates [13]. However, deviation in the dot dimension increases rapidly as the deposition time increases [12]. Meanwhile, the improvement of the island uniformity is also an important subject. It is realized that the understanding of the growth mode in heteroepitaxy can give more insights into this in situ growth technique. During the studies of the island formation of $\operatorname{In}_{x} \mathrm{Ga}_{1-x}$ As epilayers grown on (100) GaAs with $x$ varying from 0.28 to 1 [15], we have found that under a highly strained epitaxial condition, the different degree of cation ( $\mathrm{Ga}$ and $\mathrm{In}$ ) intermixing in the alloy composition can result in different island nucleation sources and then limit the island size. In this paper, we show that during the in situ formation of quantum dots, the dot uniformity can be much improved and the dot size can be tuned within a wide range, by only adjusting the alloy composition.

\section{Experimental}

All the samples were grown on undoped (100) GaAs substrates in a VG V80H MK II MBE system. The growth details have been described previously [15]. After a $200 \mathrm{~nm}$ thick GaAs buffer was grown at $580^{\circ} \mathrm{C}$, the InGaAs epilayer was then deposited to form islands. $15 \mathrm{ML}$ of $\mathrm{In}_{x} \mathrm{Ga}_{1-x}$ As with $x=1$, 0.75 , and 0.5 were deposited. Since the indium atoms tend to aggregate and desorb at higher growth temperature, so in this experiment, the InGaAs layer with a higher In composition is grown at a lower temperature, i.e., $510^{\circ} \mathrm{C}$ for $x=0.5,500^{\circ} \mathrm{C}$ for $x=$
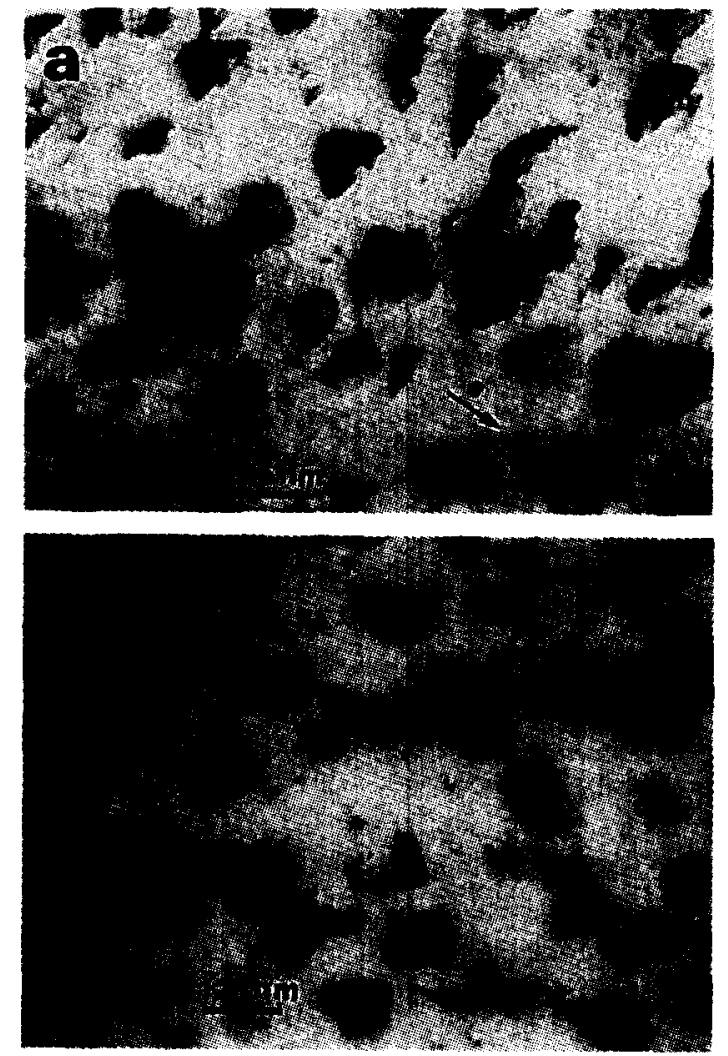

Fig. 1. Plan-view TEM images of (a) 15 and (b) $10 \mathrm{ML}$ thick InAs grown on GaAs which form islands. (a) The two-beam dark-field $(0 \overline{2} 2)$ image, and (b) the axial bright-field image.

$0.75,470$ to $480^{\circ} \mathrm{C}$ for $x=1$. The growth gate is kept at the 1.5-3 s/ML range. At the same time, 10 ML thick InAs islands were also grown. The reflection high energy electron diffraction (RHEED) patterns show very specular spots after the deposition in all cases.

\section{Results and discussion}

Figs. 1a and $1 \mathrm{~b}$ display the plan-view transmission electron microscope (TEM) images of 15 and $10 \mathrm{ML}$ thick InAs islands, respectively. For the 15 ML thick case (Fig. 1a), the two-beam dark-field image indicates that large irregular-shaped islands in the $90 \times 70-125 \times 100 \mathrm{~nm}^{2}$ range with wide separations appeared. These islands have clear (100) flat top surfaces and $\{111\}$ facets, and the density is 
about $5 \times 10^{9} \mathrm{~cm}^{-2}$. The uniformity of the islands in size and separation is poor, and coalescence of islands was observed. As the deposition thickness decreases to $10 \mathrm{ML}$ (Fig. 1b), the bright-field image indicates that the island coalescence phenomenon is suppressed and the island shape seems more regular. Some small dots $\left(25 \times 25 \mathrm{~nm}^{2}\right)$ appeared, like InP islands grown on GaAs [14]. It is important to note that the island features of the 10 and $15 \mathrm{ML}$ cases are basically similar, uniform and small InAs islands were obtained only when the deposited thickness is less than $4 \mathrm{ML}$ [12]. The plan-view TEM image of $15 \mathrm{ML}$ thick $\mathrm{In}_{0.75} \mathrm{Ga}_{0.25}$ As islands is shown in Fig. 2. It is very clear that island nucleation sites increase and islands become more regular in shape and uniform in size than those of the InAs case under the same thickness (Fig. 1a). Meanwhile, the average island size becomes smaller, about $90 \times 60 \mathrm{~nm}^{2}$, and the density is about $1 \times 10^{10} \mathrm{~cm}^{-2}$. Continuously changing the composition to $\mathrm{In}_{0.5} \mathrm{Ga}_{0.5} \mathrm{As}$, as shown in Fig. 3, islands with a density of $5 \times 10^{10} \mathrm{~cm}^{-2}$ appear; these islands, except a few coalesced ones, have a regular shape and unclear facets. The uniformity of all the island shape, the size, and the separation are much improved. Most importantly, the resultant island size is reduced to a very small value, about $28 \times 30 \mathrm{~nm}^{2}$.

The above results indicate that during the in situ formation of quantum dots, the adjustment of the alloy composition can systematically tune the island

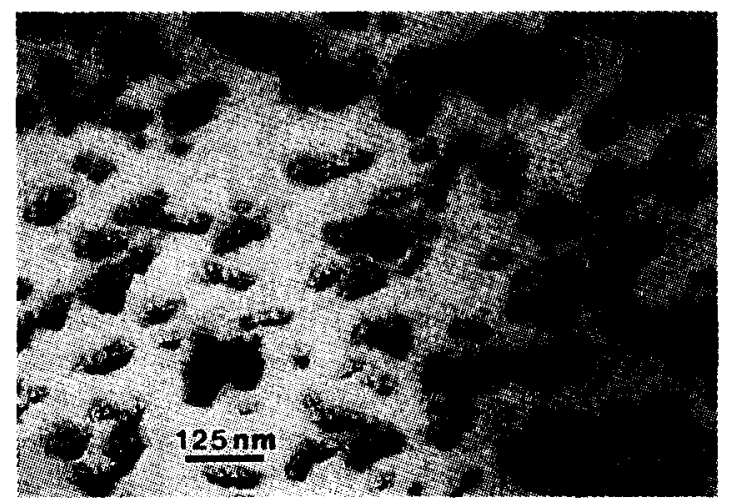

Fig. 2. The plan-view, axial bright-field TEM image of $15 \mathrm{ML}$ thick $\operatorname{In}_{x} \mathrm{Ga}_{1-x}$ As with $x=0.75$ grown on GaAs which form islands.

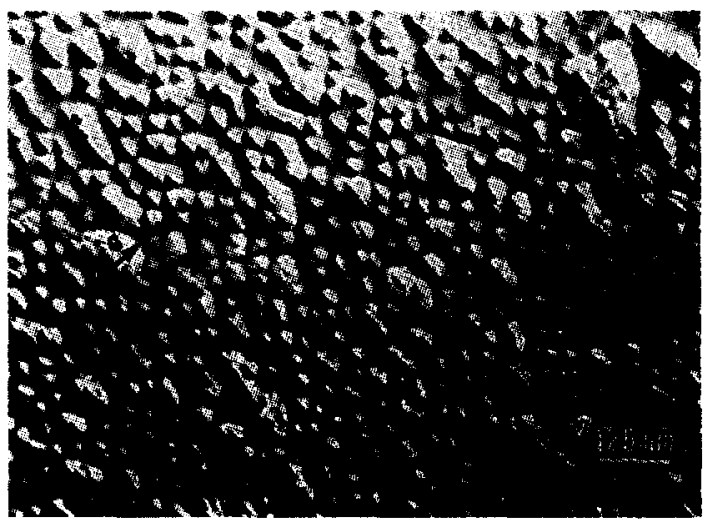

Fig. 3. The plan-view, two-beam dark-field (02̄2) TEM image of 15 ML thick $\ln _{x} \mathrm{Ga}_{1-x}$ As with $x=0.5$ grown on $\mathrm{GaAs}$ which form islands.

dimension and improve the island uniformity. The island formation mechanism in a mismatched system is very complicated and we have found that when the lattice-mismatch induced strain is large in the growth stage, the degree of the intermixing of cations ( $\mathrm{Ga}$ and In atoms) in the alloy composition becomes an important factor in determining the island nucleation sites [15]. This concept is then adopted in the fabrication of quantum dots, as illustrated in Figs. 1 to 3. It is important to note that even as the deposition thickness is increased, the $\operatorname{In}_{0.5} \mathrm{Ga}_{0.5}$ As islands still exhibit similar features [16] as those observed in Fig. 3. A similar phenomenon has already been observed in Fig. 1 that the island features for the InAs case with different thicknesses indeed look similar. So the growth kinetics rather than the deposition time determine the dot properties. The $\operatorname{In}_{x} \mathrm{Ga}_{1-x}$ As dot dimension decreases from $125 \times 100$ to $30 \times 28 \mathrm{~nm}^{2}$, when $x$ decreases from 1 to 0.5 . At the $x=0.5$ case, since the existence of highly dense island nucleation sites, the island size is constrained. Islands generally become more regular, i.e., rounded pyramids, when they grow smaller (see Ref. [12] and Figs. 1 to 3), so, as the cation-disorder limits the island size, the island uniformity is improved simultaneously.

During the fabrication of these nano-structures, the avoidance of defect generation is very important. Generally, islands in a lattice-mismatched system with larger volumes prefer to generate dislocations $[17,18]$. In this experiment, moire fringes appeared in 
the InAs and $\mathrm{In}_{0.75} \mathrm{Ga}_{0.25}$ As but not the $\mathrm{In}_{0.5} \mathrm{Ga}_{0.5}$ As dots (except in a few coalesced ones), indicating that the former dots were at least partially relaxed and contain dislocations, while the latter ones are dislocation-free. It is reasonable since InAs has a large lattice mismatch with respect to GaAs and the resultant InAs dots also have the largest dimension. So, correctly utilizing the cation-disorder phenomenon such as in the $\mathrm{In}_{0.5} \mathrm{Ga}_{0.5}$ As case can result in highquality and small-sized dots. For InAs grown on $\mathrm{GaAs}$, although the growth kinetics does not favor small-sized islands, reduction in the deposition time can still decrease the dot size [12], which suppresses the island coalescence and thus increases the uniformity (see Fig. 1).

\section{Conclusions}

In conclusion, the use of the island formation during the epitaxy of $\operatorname{In}_{x} \mathrm{Ga}_{1-x}$ As on GaAs can be applied to the in situ formation of quantum dots. Adjusting the alloy composition towards a larger degree of cation disorder can decrease the island size and improve the island uniformity simultaneously. For the same deposition thickness, $\mathrm{In}_{0.5} \mathrm{Ga}_{0.5}$ As dots reach the minimum value in size, i.e., $30 \times 28 \mathrm{~nm}^{2}$, and exhibit the best uniformity. The small-sized dots also prevent the introduction of dislocations, favored by the formation of high-quality quantum dots.

\section{Acknowledgements}

This work was supported by the National Science Council of the Republic of China under contract no. NSC83-0404-E002-014.

\section{References}

[1] H. Temkin, G.J. Dolan, M.B. Panish and S.N.G. Chu, Appl. Phys. Lett. 50 (1987) 413.

[2] J.A. Lebens, C.S. Tsai and K.J. Vahala, Appl. Phys. Lett. 56 (1990) 2642.

[3] Y. Nagamune, S. Tsukamoto, M. Nishioka and Y. Arakawa, J. Cryst. Growth 126 (1993) 707.

[4] I.H. Tan, R. Mirin, V. Jayaraman, S. Shi, E. Hu and J. Bowers, Appl. Phys. Lett. 61 (1993) 300.

[5] T. Chikyow and N. Koguchi, in: Extended Abstracts of Int. Conf. on Solid State Devices and Materials, Chiba, 1993, p. 350.

[6] R. Nötzel, J. Temmyo, H. Kamada, T. Furtua and T. Tamamura, Appl. Phys. Lett. 65 (1994) 457.

[7] R. Hull and J.C. Bean, in: Strain-Layer Superlattices: Materials Science and Technology, Semiconductors and Semimetals, Vol. 33, Ed. T.P. Pearsall (Academic Press, New York, 1991) ch. 1.

[8] D.J. Eaglesham and M. Cerullo, Phys. Rev. Lett. 64 (1990) 1943.

[9] S. Guha, A. Maduhkar and K.C. Rajkumar, Appl. Phys. Lett. 57 (1990) 2110.

[10] C.W. Snyder, B.G. Orr, D. Kessler and L.M. Sander, Phys. Rev. Lett. 66 (1991) 3032.

[11] D. Leonard, M. Krishnamurthy, C.M. Reaves, S.P. Denbaars and P.M. Petroff, Appl. Phys. Lett. 63 (1993) 3203.

[12] J.M. Moison, F. Houzay, F. Barthe, L. Leprince, E. Andrá and O. Vatel, Appl. Phys. Lett. 64 (1994) 196.

[13] J. Ahopelto, A.A. Yamaguchi, K. Nishi, A. Usui, H. Sakaki and Y. Mochizuki, in: Extended Abstracts of Int. Conf. on Solid State Devices and Materials, Tsukuba, 1992, p. 281.

[14] C.M. Reaves, V. Bressler-Hill, M. Krishnamurthy, S. Varma, P.M. Petroff, W.H. Weinberg, and S.P. Denbaars, in: Proc. 6th Int. Conf. on Indium Phosphide and Related Materials, Santa Barbara, CA, 1994, p. 611.

[15] S.Z. Chang, T.C. Chang and S.C. Lee, J. Appl. Phys. 73 (1993) 4916.

[16] J.Y. Yao, T.G. Andersson and G.L. Dunlop, J. Appl. Phys. 69 (1991) 2224.

[17] W.A. Jesser and J.H. van der Merwe, Surf. Sci. 31 (1972) 229.

[18] M. Lentzen, D. Gerthsen, A. Förster and K. Urban, Appl. Phys. Lett. 60 (1992) 74. 